\title{
A novel experimental method for the in situ detection of thermal bridges in building envelopes based on active infrared thermography and singular value decomposition analysis
}

by Rémy Douguet*, Thanh-Tung Ha*, Vincent Feuillet**, Johann Meulemans* and Laurent Ibos**

* Saint-Gobain Recherche, 93303 Aubervilliers Cedex, France, johann.meulemans@saint-gobain.com

** CERTES/OSU Efluve, Univ. Paris-Est, 94010 Créteil Cedex, France, vincent.feuillet@u-pec.fr, ibos@u-pec.fr

\begin{abstract}
A novel experimental method for the detection of thermal bridges in building envelopes is presented. The combination of active infrared thermography (IRT) and singular value decomposition (SVD) allows to detect the spatial variations of insulation of the inspected area. The ambient air temperature is modulated at night and the region of interest is monitored with an infrared camera. A SVD analysis is then applied on the recorded thermographic sequence. The capability of the method to detect in situ thermal bridges was demonstrated at room-scale for different wall configurations and different seasons with a low-cost infrared camera.
\end{abstract}

\section{Introduction}

Multi-layer walls with high thermal resistance are widely used to reduce heat losses in buildings. Nevertheless, the presence of thermal bridges tends to reduce their performance [1]. Different types of thermal bridges can be found in a building, namely geometrical and structural thermal bridges. Depending on the evaluation method used, thermal bridges can represent $11 \%$ to $30 \%$ of the energy final demand $[2,3,4,5]$. Thermal bridges may also have a detrimental impact on the thermal comfort of the occupants (degradation of air quality due to undesired condensation and mould growth [6]). It is thus extremely important to use a method (or a set of methods) to detect the presence of thermal bridges.

The use of infrared thermography (IRT) for building applications/inspections was extensively documented in the past decades (e.g., see [7,8,9] and references therein). There are two ways to use an infrared camera [10]. The first approach involves the recording of a single thermal image or a sequence of thermal images. This is commonly termed passive thermography, as the thermographer observes the scene without intervening. In the case of a building, this kind of approach requires a significant temperature difference between the interior and the exterior, which limits the testing opportunities to only certain periods of the year or the day. The second approach, called active thermography, is a common practice in the field of thermal non-destructive evaluation (TNDE) of materials. A material is stimulated (e.g., by a photothermal source) and the thermal response of the material (i.e., the thermogram) indicates whether or not a defect (or flaw) is present in the material. In the case of a building, it consists in heating a wall on one of its faces and using an infrared camera to record the temperature evolution. The advantage of the active approach is that a test can be carried out at any time. This method does, however, require additional equipment.

Standard ISO 13187 [11] specifies a qualitative method, by thermographic examination (infrared method), for detecting thermal irregularities in building envelopes. This passive method is used to identify wide variations in the thermal properties, including air tightness, of the components constituting the external envelopes of buildings. However, its use is often restricted to the cold season provided strict operating conditions are met (e.g., see [7-8]). Quality controls, if any, may thus be postponed. Grinzato et al. [12] compared the potential of different experimental methods based on active IRT for the detection of defects in buildings. Both natural or artificial heat sources were used (i.e., the sun or controlled radiant heat sources, respectively). The building envelope was monitored in transient regime and the thermal thermograms were analyzed to identify potential defects. Recently, a method for an automatic detection of thermal bridges in buildings was proposed by Garrido et al. [13]. This passive method requires an image processing of raw thermal images. First, an image rectification is performed to correct geometrical distorsion of images. Then, a median filter is used increase the signal to noise ratio. This allows detecting lines corresponding to thermal bridges. False detections are reduced using a thresholding method. Three criterions are used to improve the rate of automatic detection of thermal bridges up to $55 \%$.

In some limited experimental conditions, thermal bridges can be detected from a sequence of thermal images without any post-processing. For instance, thanks to optimal weather conditions, thermal bridges between the floors and the exterior walls of a building can be easily detected. Conversely, the thermal contrast induced by the presence of integrated thermal bridges in a building wall is generally quite low (i.e., smaller than $1 \mathrm{~K}$ ). Thus, the detection of these thermal bridges from raw images requires the use of an infrared camera with a high signal to noise ratio (e.g., a camera equipped with a cooled detector). For thermographic surveys, the use of micro-bolometer cameras is more relevant due to the reduced price of these cameras. Thus, in that case, a post-processing of thermal images should be done in order 
to improve the signal to noise ratio and thus to perform an accurate detection of integrated thermal bridges of a building wall.

There is a need to develop new methods for the systematic detection of thermal bridges in buildings. These methods should be especially less sensitive to the external environment and potentially used all year long. In this work, active IRT is used to perform an in situ detection of integrated thermal bridges within the walls of a building. The objective is to be able to perform a diagnosis whatever the season or the weather conditions, with a short test duration and a moderate applied heating power.

\section{Experimental and analysis protocol}

\subsection{Investigated building}

The investigated building is a bungalow located at Saint-Gobain Recherche (SGR) in Aubervilliers, France (see Figure 1). Two insulation configurations are studied. A schematic view of the wall corresponding to structure $A$ is presented in Figure 2(a). In this configuration, a first insulating layer consisting of Vacuum Insulation Panels (VIP) of standard dimensions is placed on the walls $(20 \mathrm{~mm})$, floor and ceiling $(30 \mathrm{~mm})$ made of polyurethane (PU) with remaining space filled with expanded polystyrene (EPS). A second insulation layer of $28 \mathrm{~mm}$ of EPS is added. In the second configuration (B), a standard insulation with glass wool (GW) is investigated (see Figure 2(b)). In both cases, standard gypsum plasterboards (PB) and oriented strand boards are placed on the walls/ceiling and the floor, respectively.
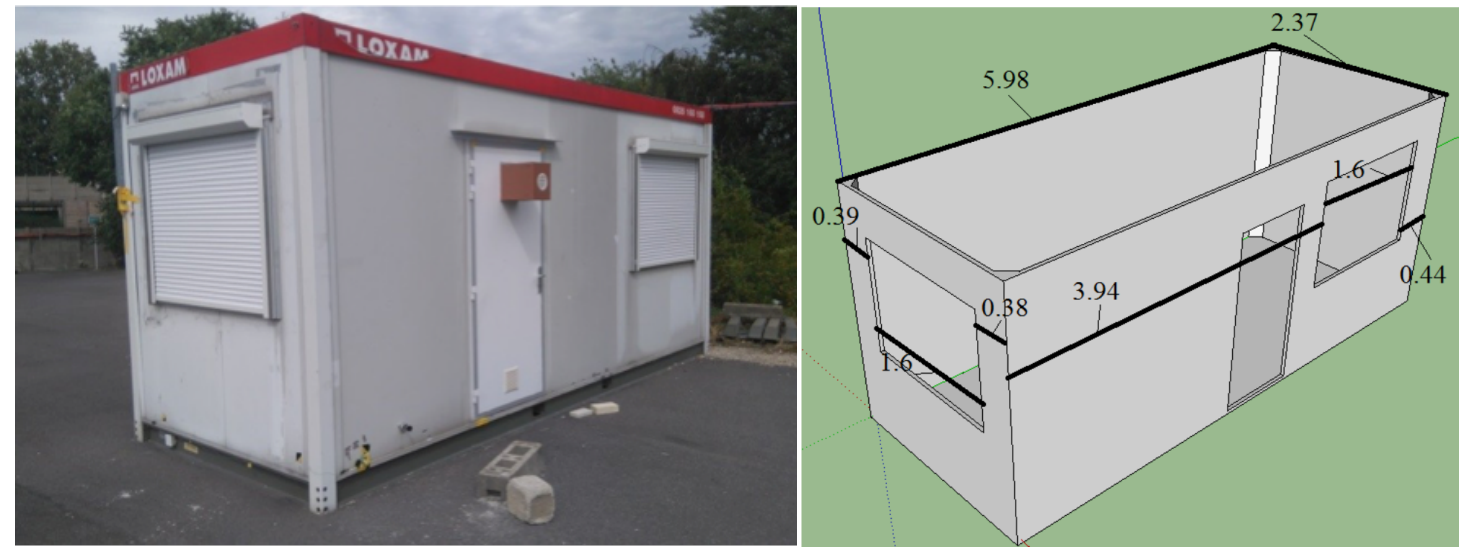

Fig. 1. Picture of the investigated bungalow (left) and its dimensions in meter (right)
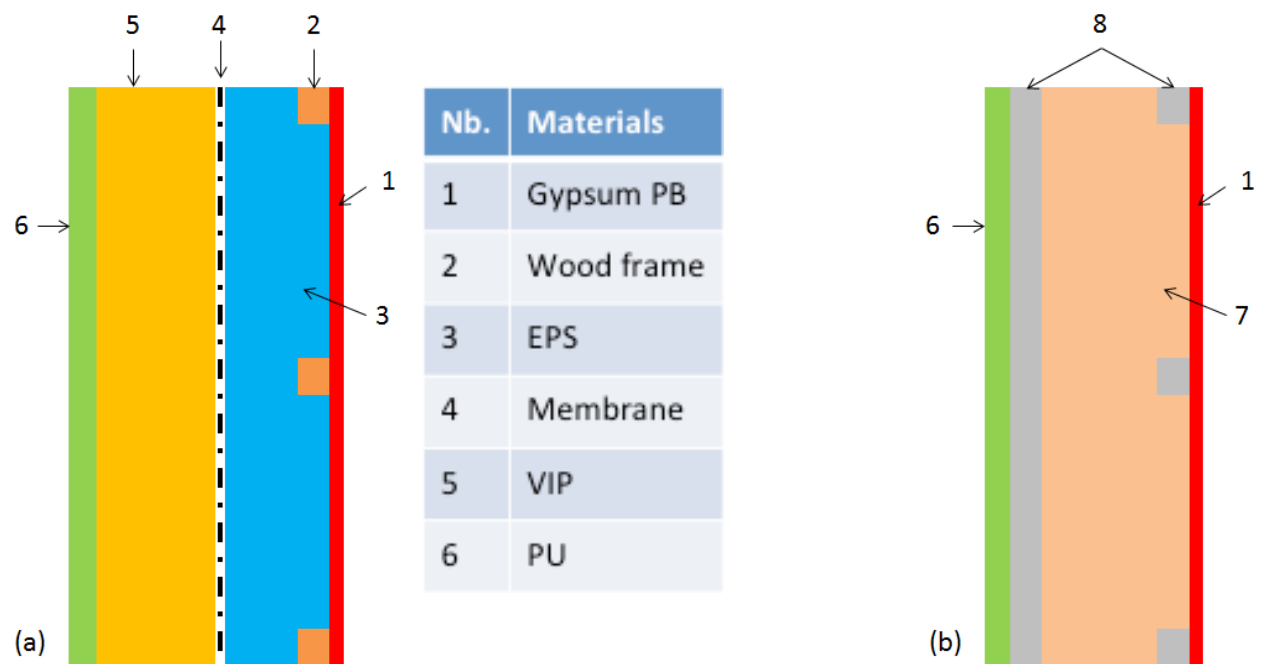

\section{Nb. Materials}

$1 \quad 1 \quad$ Gypsum PB

\section{Gypsum PB}

2 Wood frame

3 EPS

4 Membrane

5 VIP

$6 \quad \mathrm{PU}$

Fig. 2. Schematic views of the investigated walls: (a) configuration $A$, (b) configuration $B$ 


\subsection{Experimental protocol}

An indoor view of the in situ experimental test bench is presented in Figure 3. Several sensors located inside and outside the building measure continuously a set of physical parameters: wall temperatures measured by means of two infrared cameras (long wave cooled FLIR SC7300 ${ }^{\mathrm{TM}}$ and uncooled FLIR A35 ${ }^{\mathrm{TM}}$ ), interior air temperature measured by K-type thermocouples and exterior air temperature measured with a weather station. The experimental protocol is controlled by a program developed under LabVIEW ${ }^{\mathrm{TM}}$.

Noise reduction is achieved by averaging, every 30 seconds, 100 infrared images recorded at $50 \mathrm{~Hz}$. The average image contains digital levels which represent incoming luminance from object to camera. The numerical values can be converted to temperatures by applying Planck's law and by taking into account environmental conditions and surface emissivity. In this study, we only focus on calculations with digital levels. As the objective is to develop an experimental protocol to detect the presence of thermal bridges, the conversion of digital levels to temperature data is not required [14].

In order to implement an active approach, the room is heated with electrical heaters with variable power (e.g., rolled heating mats or fan heaters). They are placed on the floor so as to obtain a homogeneous interior air temperature and that they are not visible on the IR images. Several measurement campaigns of about 10 consecutive days each were performed at different seasons (i.e., autumn/winter, mid-season and summer) in order to check the possibility to detect thermal bridges whatever the weather conditions. The room was submitted to successive thermal loads during the nights (in order to avoid perturbations due to solar radiation on the test bench walls), with heating and cooling phases (square signals) varying from 10 to 120 minutes and a heating power ranging from 250 to $1200 \mathrm{~W}$ (see Figure 4). Increases of air and wall surface temperatures were typically around $10^{\circ} \mathrm{C}$ and $5^{\circ} \mathrm{C}$ respectively, for a two-hour heating phase and a heating power of $1200 \mathrm{~W}$. In this study, we investigated the influence of heating power and the duration of the heating and the cooling phases on the detection of irregularities/defects within each wall configuration. For the sake of brevity, only results obtained for equal durations of both heating and free cooling phases will be presented in this paper.

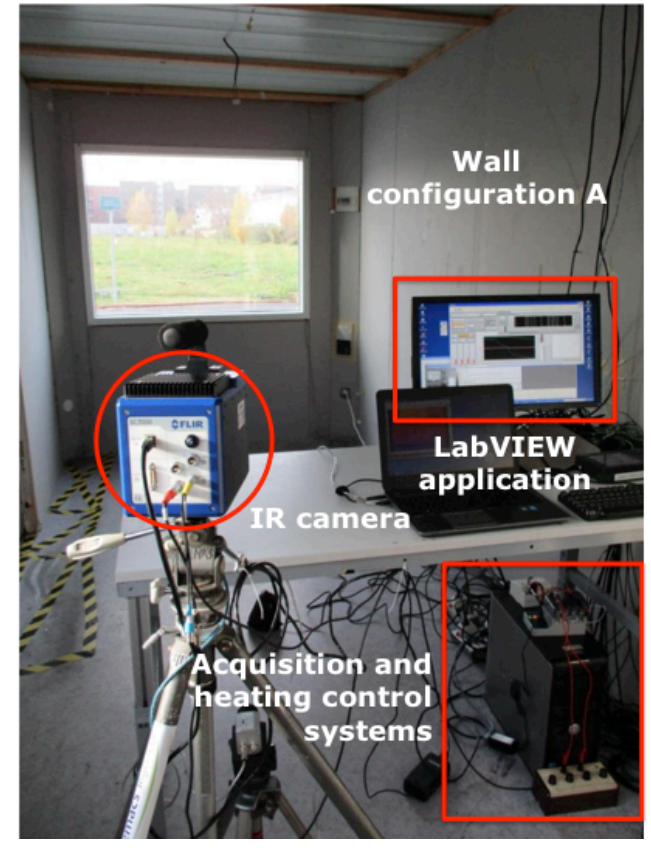

Fig. 3. View of the indoor experimental set-up

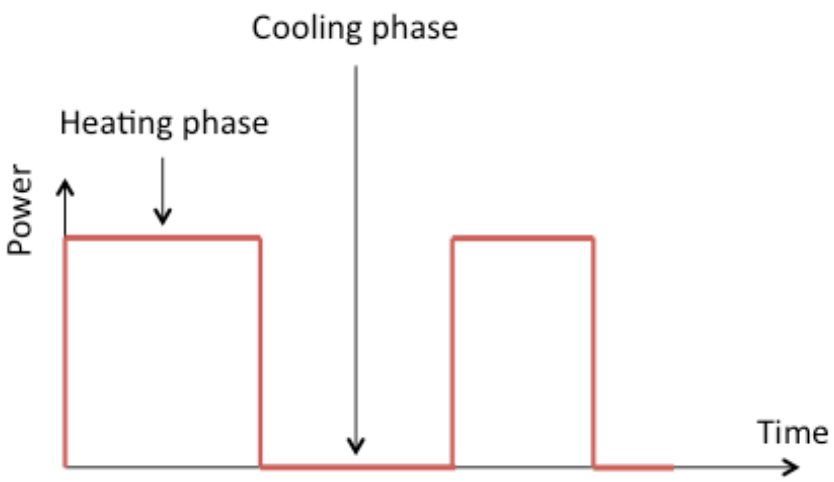

Fig. 4. Example of square signal used as heating and cooling phases

\subsection{Image processing}

In this work, the use of Singular Value Decomposition (SVD) analysis is investigated to enhance the detection of thermal bridges on the interior surface of the wall. SVD is an interesting tool for the extraction of the spatial and temporal information from a thermographic sequence in a compact and simplified manner. The SVD of an $m \times n$ matrix $X$ is a linear algebraic factorization which can be calculated as follows $[15,16,17]$ :

$$
\boldsymbol{X}=\boldsymbol{U} \boldsymbol{S} \boldsymbol{V}^{t}
$$


where $U$ is an $m \times m$ orthogonal matrix, $S$ is a $m \times n$ diagonal matrix (with the singular values of $X$ in the diagonal, sorted in descending order) and $V^{t}$ is the transpose of an $n \times n$ orthogonal matrix:

$$
\left(\begin{array}{ccc} 
& \boldsymbol{X} & \\
x_{11} & \cdots & x_{1 n} \\
\vdots & \ddots & \\
x_{m 1} & & x_{m n}
\end{array}\right)=\left(\begin{array}{ccc} 
& \boldsymbol{U} & \\
u_{11} & \cdots & u_{1 m} \\
\vdots & \ddots & \\
u_{M 1} & & u_{m m}
\end{array}\right)\left(\begin{array}{ccc} 
& \boldsymbol{S} \\
s_{11} & 0 & \cdots \\
0 & \ddots & \\
\vdots & & s_{m n}
\end{array}\right)\left(\begin{array}{ccc} 
& \boldsymbol{V}^{t} \\
v_{11} & \cdots & v_{1 n} \\
\vdots & \ddots & \\
v_{n 1} & & v_{n n}
\end{array}\right)
$$

The thermographic sequence must be rearranged so that the columns of matrix $X$ correspond to the thermograms at each time (see Figure 5):

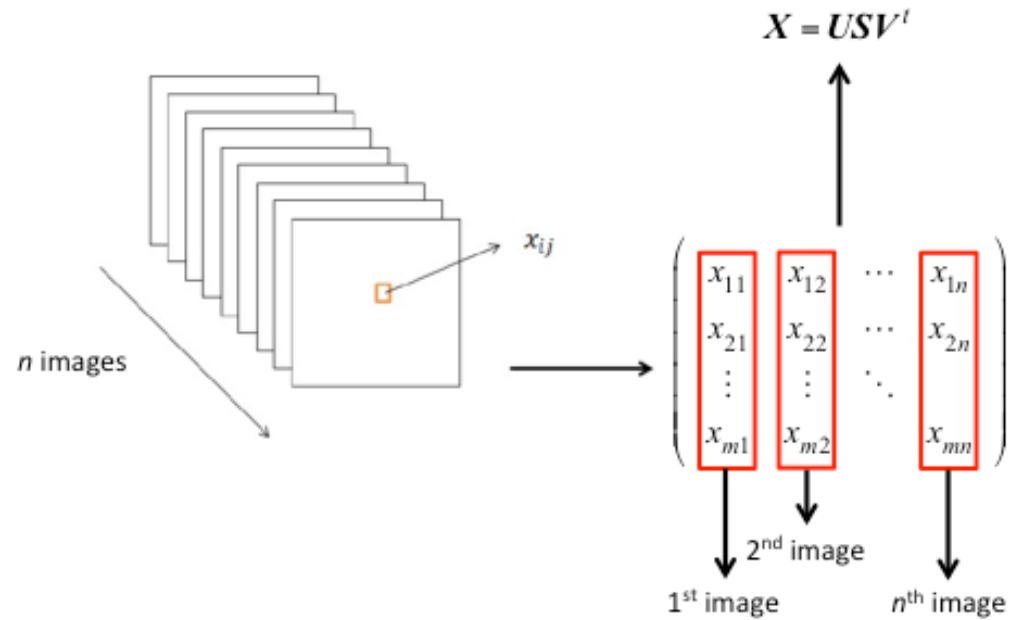

Fig. 5. Rearrangement of the thermographic sequence before application of the SVD treatment

After applying the SVD on matrix $X$, the columns of $U$ represent a set of orthogonal statistical modes known as Empirical Orthogonal Functions (EOF), which describe spatial variations of data. On the other hand, the Principal Components (PC), which represent time variations, are arranged row-wise in matrix $V^{t}$. The first EOF will represent the most characteristic variability of the data, the second EOF (denoted further as EOF $\mathrm{n}^{\circ} 2$ ) will contain the second most important variability, and so on. Usually, original data can be adequately represented with only a few EOFs.

The other advantage of SVD is that it can reduce noise from original sequence by removing high orders of singular vectors. Superior orders contain less important information, so if some vectors belong to high enough orders, they can be considered as noise. A reconstruction of the thermographic sequence (inverse SVD) from the truncated SVD matrices leads to a filtered sequence. Moreover, the increase of signal to noise ratio induced by an SVD processing allows then to use classical image segmentation methods (such as gradient for instance) to extract edges of thermal irregularities.

\section{Results}

In Figure 6, we represent the first three EOFs and their associated PCs extracted from a sequence of infrared images obtained using a micro-bolometer camera (FLIR A35 ${ }^{\mathrm{TM}}$ ) for the wall configuration $A$ and a north façade. In this case, measurements were made in autumn with 120 minutes of heating phase and 120 minutes of cooling phase $(120 / 120)$ for a heating power load of $1170 \mathrm{~W}$. Integrated thermal bridges are easily detected using this experimental protocol on the whole surface of the observed wall.

Figure 7 shows EOF $\mathrm{n}^{\circ} 2$ for the wall configuration $\mathrm{B}$ and an east façade obtained in summer conditions with a cooled IR camera (FLIR SC7300 ${ }^{\mathrm{TM}}$ ) for the same heating and cooling phase durations and heating power. Here a gradient scan has been applied in addition to the SVD. The presence of the structural thermal bridge is also clearly seen in this picture.

The proposed active approach can thus be used to localize the position of a thermal bridge without any prior knowledge on its properties or position. Besides, these first results proved the ability of the method to detect thermal bridges whatever the measurement conditions: season, thermal camera, composition and orientation of the wall. 

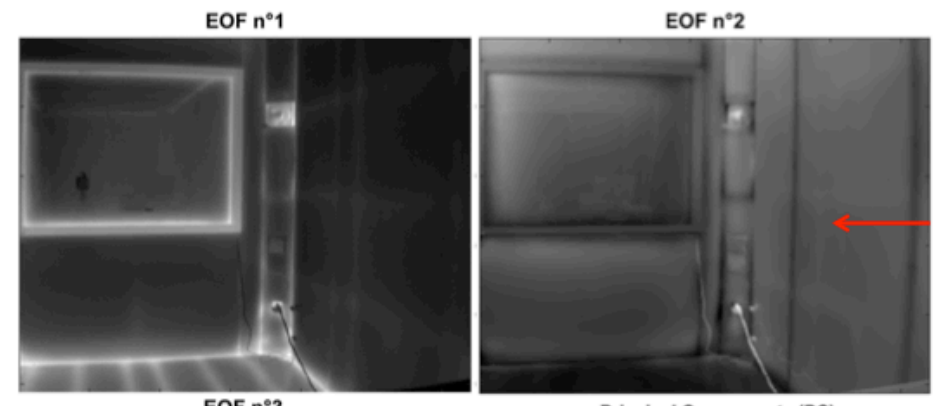

Wall configuration A
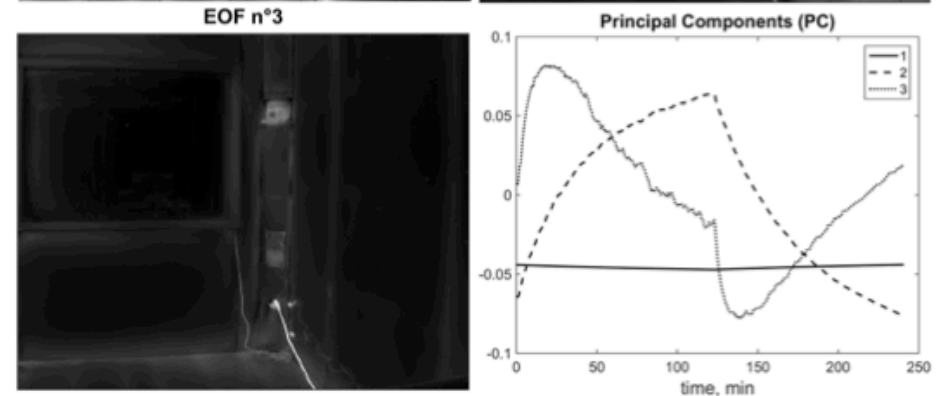

Fig. 6. EOFs $n^{\circ} 1$ to 3 and their associated Principal Components for wall configuration $A$ (autumn conditions; uncooled micro-bolometer IR camera; 120/120 minutes of heating and cooling phases; $1170 \mathrm{~W}$ )

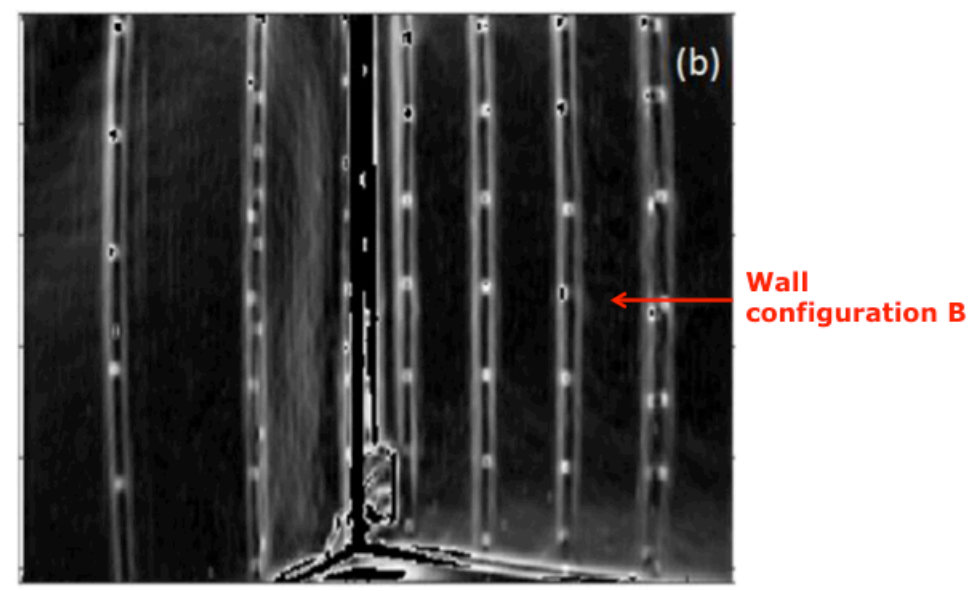

Fig. 7. EOF $n^{\circ} 2$ with gradient scan for wall configuration $B$

(summer conditions; cooled IR camera; 120/120 minutes of heating and cooling phases; $1170 \mathrm{~W}$ )

It is now interesting to compare these results to those obtained without artificially heating the room (passive infrared thermography). Figure 8 shows the digital levels obtained for the wall configuration A during a thermographic survey conducted in accordance with standard ISO 13187. One can distinguish a piece of integrated thermal bridge for the configuration $\mathrm{A}$ but the detection is quite limited. The conditions of application of a passive thermography control were not met for the wall configuration $B$ (very hot summer period).

Different heating and cooling phase durations were also investigated. Figure 9 represents EOF $n^{\circ} 1$ for the wall configuration A in the same configuration as in Figure 6 but the heating and cooling phases vary from 20/20 to 120/120. The images obtained are very similar between the different durations. So a quite short experiment duration (i.e., less than one hour) is sufficient to obtain a satisfactory detection.

Finally, the influence of the heating power is studied in Figure 10 . Here are presented EOF $n^{\circ} 2$ for the wall configuration A for two heating power levels $(670 \mathrm{~W}$ and $1170 \mathrm{~W})$ for the same heating and cooling phase durations. The use of an important heating power shows a better detection of thermal bridges. A better contrast on irregularities is observed, i.e. the signal to noise ratio is improved. 


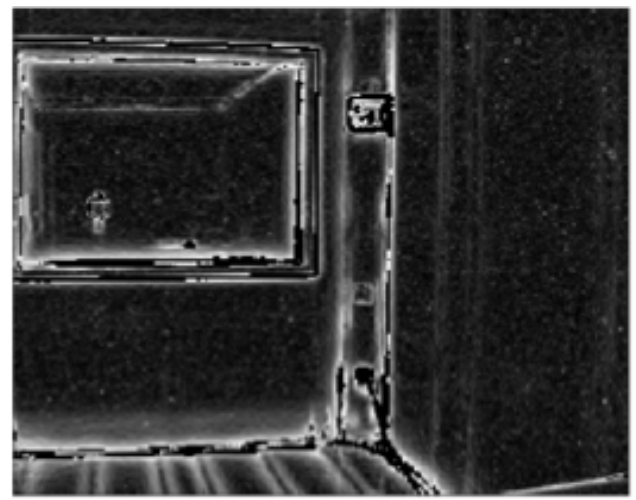

Fig. 8. Digital data obtained for wall configurations $A$ in autumn during a passive experiment

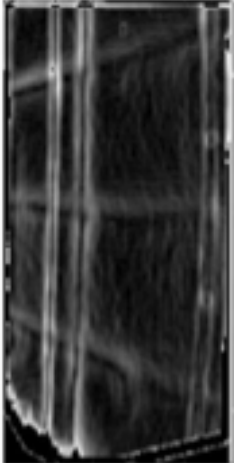

$20 / 20$

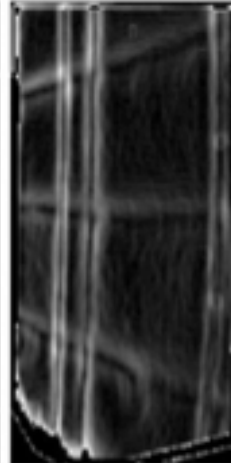

$60 / 60$

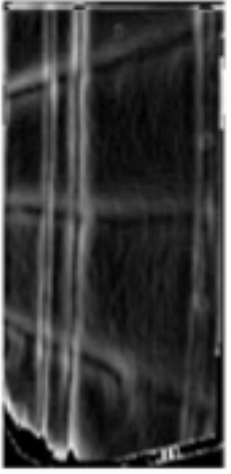

$80 / 80$

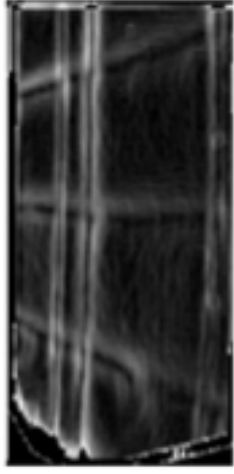

$120 / 120$

Fig. 9. EOF $n^{\circ} 1$ for wall configuration A for different heating/cooling phase durations (autumn conditions; uncooled micro-bolometer IR camera; $1170 \mathrm{~W}$ )

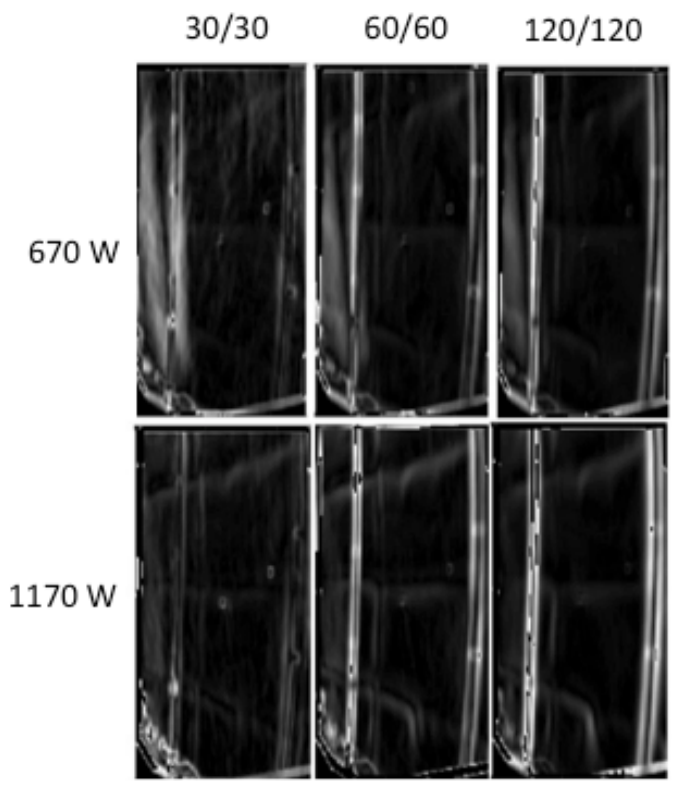

Fig. 10. EOF $n^{\circ} 2$ for wall configuration A for different heating powers and heating/cooling phase durations (autumn conditions; uncooled micro-bolometer IR camera) 


\section{Conclusion}

The association of active infrared thermography with singular value decomposition analysis made it possible to carry out in situ detection of thermal bridges. The active method proposed enhanced the signal to noise ratio and consequently allowed an easier detection of integrated thermal bridges even using a low-cost micro-bolometer infrared camera. It did not require the conversion of the digital data measured by the camera into temperature values. In the experimental situation considered, the emissivity of the observed surface was not spatially varying and the environment could be considered as uniform. In that case, as expected, the conversion of digital levels into temperature data is not required for the detection of thermal irregularities using a sequence of thermal images.

The detection of thermal bridges in an extended zone was possible thanks to the active method chosen, i.e. heating of the ambient air inside the building. Indeed, a rather uniform thermal excitation of the wall surface is achieved. A limited increase of air temperature of 5 to $10 \mathrm{~K}$ is sufficient to perform such analysis. Moreover, this protocol does not require any prior information on the wall structure or possible location of thermal bridges. It has been shown that the detection of this type of integrated thermal bridges was possible whatever the season (winter, mid-season or even summer conditions). Finally, we observed that SVD analysis might bring information on the choice of experimental parameters, for instance the heating and cooling phase durations and the heating power of the active experiment.

Further works will focus on the comparison between experimental data and numerical simulation results. The final step of the study will consist in using a parameter estimation method in order to fit experimental data with a thermal model by adjusting some modelling parameters, especially the thermal properties of thermal bridges.

\section{REFERENCES}

[1] Farkh S., "Les ponts thermiques dans le bâtiment : Mieux les connaître pour mieux les traiter" (in French). Technical report, Centre Scientifique et Technique du Bâtiment (CSTB), 2014.

[2] Theodosiou T.G., Papadopoulos A.M., The impact of thermal bridges on the energy demand of buildings with double brick wall constructions, Energy and Buildings. - Vol. 40, no 0 (2008), pp. 2083-2089, 2008.

[3] Spiekman M., "Contribution to the asiepi project: summary of a dutch study on the quantification of thermal bridge effects on the energy performance". Report, Summary report of ASIEPI WP4, 2010.

[4] Centre Scientifique et Thermique du Bâtiment. "Réglementation Thermique" (in French). Ed. CSTB, Paris, France, 2000.

[5] Capozzoli A., Gorrino A., Corrado V., A building thermal bridges sensitivity analysis, Applied Energy. - Vol. 107 (2013), pp. 229-243, 2013.

[6] Larbi A. B., Statistical modelling of heat transfer for thermal bridges of buildings, Energy and Buildings. - Vol. 37, no 0 (2005), pp. 945-951, 2005.

[7] Balaras C. A., Argiriou A. A., Infrared thermography for building diagnostics, Energy and Buildings. - Vol. 34, no 2 (2002), pp. 171-183, 2002.

[8] Kylili A., Fokaides P. A., Christou P., Kalogirou S. A., Infrared thermography (IRT) applications for building diagnostics: A review, Applied Energy. - Vol. 134 (2014), pp. 531-549, 2014.

[9] Pajani D., "La thermographie du bâtiment : Principes et applications du diagnostic thermographique" (in French). Eyrolles, 2012.

[10] Maldague X., "Theory and practice of infrared technology for non-destructive testing". Ed. John-Wiley \& Sons, 2001.

[11] British Standards Institution, "Thermal performance of buildings - Qualitative detection of thermal irregularities in building envelopes - Infrared method”. BS EN 13187:1999, London (United Kingdom), 1999.

[12] Grinzato E., Vavilov V., Kauppinen T., Quantitative infrared thermography in buildings, Energy and Buildings. Vol. 29, no 1 (1998), pp. 1-9, 1998.

[13] Garrido I., Lagüela S., Arias T., Balado J., Thermal-based analysis for the automatic detection and characterization of thermal bridges in buildings, Energy and Buildings. - Vol. 158 (2018), pp. 1358-1367, 2018.

[14] Marchetti M., Ludwig S., Dumoulin J., Ibos L., Mazioud A. "Active Infrared Thermography for Non-Destructive Control for Detection of Defects in Asphalt Pavements", proceedings of 9th Quantitative InfraRed Thermography conference, paper QIRT2008-05_05_08 Krakow (Poland), 2008.

[15] Rajic N., Principal component thermography for flaw contrast enhancement and flaw depth characterization in composite structures, Composite Structures. - Vol. 58 (2002), pp. 521-528, 2002.

[16] Vrabie V., Perrin E., Bodnar J.-L., Mouhoubi K., Detalle V. "Active IR thermography processing based on higher order statistics for nondestructive evaluation", proceedings of the 20th European Signal Processing Conference (EUSIPCO), pp. 894-898 Bucharest (Romania), 2012.

[17] Dumoulin J., Ibos L., Ibarra-Castanedo C., Mazioud A., Marchetti M., Maldague X., Bendada A., Active infrared thermography applied to defect detection and characterization on asphalt pavement samples: comparison between experiments and numerical simulations, Journal of Modern Optics. - Special Issue on Advanced Infrared Technology and Applications, Vol. 57, no 18 (2010), pp. 1759-1769, 2010. 